\title{
Effect of Women's Autonomy on Neonatal Mortality and Associated Factors in Ethiopia: A Cross- Sectional Population-Based Study
}

Habtamu Dessie ( $\square$ habtamudessie54@gmail.com )

Injibara University

\section{Research Article}

Keywords: Women's Autonomy, Neonate, Mortality, Ethiopia

Posted Date: January 18th, 2021

DOI: https://doi.org/10.21203/rs.3.rs-135763/v1

License: (c) (1) This work is licensed under a Creative Commons Attribution 4.0 International License.

Read Full License

Version of Record: A version of this preprint was published at BMC Women's Health on April 16th, 2021. See the published version at https://doi.org/10.1186/s12905-021-01308-2. 
1 Effect of Women's autonomy on neonatal mortality and associated factors in

\section{Ethiopia: A cross sectional population based study}

3 Habtamu Dessie Mitiku

4 Department of Statistics, College of Natural and Computational Science, Injibara University, Injibara,

5 Amhara, Ethiopia

$6 \quad$ Email:- habtamudessie54@gmail.com

7

8 Abstract

9 Introduction: Women's decision-making autonomy is extremely important for the development of maternal, neonatal, and child healthcare utilization. However, there's no evidence on the association of

11 women's decision-making autonomy with neonatal mortality rate at a national level in Ethiopia.

12 Therefore, this study aimed to assess the effect of women's autonomy on neonatal mortality rate and its 13 associated factors in Ethiopia.

14 Methods: A total of 5,128 neonates born 5 years before the survey from Ethiopian Demographic and 15 Health Survey 2016 were reviewed. A multivariable logistic regression model was employed to assess the 16 effect of women's autonomy and identify the determinate predictors of neonate death risk.

17 Results: The rate of neonatal mortality in Ethiopia was 20.7 per 1000 live births). Women's hadn't 18 autonomy in health care increase neonatal death by 2.72 times compared with those that had autonomy.

19 Hadn't postnatal care was caused to grown neonatal death by 5.48 times (AOR 5.48, 95\% CI: 1.29, 23.26).

20 Delivering at a health institution had 0.61 times lowered neonatal death risk compared with delivering at 21 of health institution without a health facility(AOR 0.61, 95\% CI: 0.38,0.97). Breastfeeding immediately

22 within $1 \mathrm{hr}$ after birth had 0.17 times reduce neonatal death risk compared with not initiation of 23 breastfeeding(AOR 0.17, 95\% CI: 0.12,0.26). Women's gave birth single had 0.09 times reduced neonatal 24 death risk than those that gave birth multiple (AOR 0.09, 95\% CI: 0.05,0.18). Unknowingly, male 25 neonates had a 1.84 times higher risk of death than females (AOR 1.84, 95\%CI: 1.20,2.81). 
26 Conclusion: Neonatal mortality rate was significantly related to women's hadn't deciding power on health

27 care, hadn't postnatal care, delivered out of health institution, breastfed not immediately, and gave birth

28 multiple. It is important to encourage mothers autonomy, use postnatal care service, and deliver in health

29 institutions.

30 Keywords: Women's Autonomy, Neonate, Mortality, Ethiopia

\section{$31 \quad$ Introduction}

32 Women's autonomy is the ability to access information and make decisions about one's own

33 business[1-3]. Hence, women's autonomy undoubtedly contributes to several health advantages for both

34 the mother and their children[4] and it's a priority of proceeding generations. Evidence suggests that

35 women in developing countries often have limited autonomy and control over their health regarded

36 decisions[2]. Even though, both the maternal and neonatal health provision needs a multi-sector approach

37 a robust decision-making autonomy of mother is vital for reversing back the barriers at the

38 household level[5]. Because, limited women's decision-making power delays maternal healthcare

39 utilization like antenatal care (ANC), postnatal care (PNC), and delivery health institutions[6-9]. Besides,

40 limited participation in access of health service for their on interst, lower involvement of women in

41 decision has also impact on the socio-economic, emotional, fertility decision, contraceptive use, and

42 sexual lifetime of the women[10], which results an entire population problem.

44 Studies show that the decision-making autonomy of females is low, specifically in developing countries.

45 However, scaling up the women's role in a decision leads to better uptake of healthcare access, poverty

46 reduction, and household economic growth[2, 11-16]. Indeed, in low-income countries, over women's

47 important role in the family, they essentially have seen as an ordinary homemaker. 
In Ethiopia, studies show that maternal and neonatal health coverage is low[17]. According to the 2016 Ethiopian demographic health survey (EDHS 2016) report, only 11-18\% of women were involved in

51 making decisions alone, and 66-68\% together with their husband or partner[18]. Also, the neonatal

52 mortality rate in Ethiopia was 30death per 1000 live births[19]. Most importantly the first 28 days of life

53 is the most vulnerable time for a child's survival[20]. According to UNICEF, 2020 report, the death

54 proportion of neonates globally on average is 17 death per 1000 live births in 2019, and it dropped by

$5552 \%$ from 38 deaths per 1000 in 1990. Furthermore, the report also shows that around 2.4 million

56 children died in the first month of life and closely 6,700 children died every day of these about $33.33 \%$ of

57 all neonatal deaths occurring within the first day after birth, and close to $75 \%$ occurring within the first

58 week of birth in 2019[20].

60 Despite a downing in death rate of below the age of the first 28 days after birth in the globe, marked

61 differences in death rates found vary from place to pace in the world[20]. According to the recent

62 UNICEF report, East Africa and South Asia, placed at the top in neonatal death with a projected rate of

63 twenty seven and twenty five deaths per 1,000 live births in 2019, respectively[20]. The report also

64 showed that sub-Saharan Africa neonates was ten times greater risk of death than among who was born in

65 a developed world and the death risk of neonate in the highest death country was 55 times higher than its

66 counterpart. Of these event more than 50\% of neonate deaths happened Ethiopia [20].

67

68 According to the sustainable development goal agenda of 2030 by improving maternal health care and

69 engagement of women can achieve the reduction of maternal and neonate, infant, and child mortality.

70 Hence, to achieving these targets requiring a scale-up of high impact and cost-effective maternal and

71 neonatal interventions in Ethiopia[21, 22]. Consequently, Women's empowerment in decisions is vital to

72 overcome the foremost mentioned problems. Therefore, this study aimed to assess the impact of women's

73 autonomy on neonatal mortality and associated factors in Ethiopia. 


\section{Method}

\section{Data source, sampling and data collection}

76 The data for this study was extracted from the EDHS 2016. The EDHS 2016 is the fourth and most recent

77 in the Demographic and Health Survey series in Ethiopia[18]. The survey was conducted in 9 regional

78 states and 2 city administrations of Ethiopia[18]. Further details on sampling strategy can be found in the

79 DHS manual[18]. A total of 16,650 eligible women between 15 and 49 years were approached to be

80 interviewed. A response rate of $95 \%$ was observed with 15,683 women completing the interviews. The

81 survey incorporated both women who were permanent household residents as well as visitors of the

82 household. The survey contained variety of standard questionnaires recording information on basic socio-

83 demographic information and detailed bio-medical information. This study analysis only included

84 children less than one months of age, living with an eligible respondent, in accordance with the

85 denominator of the NMR definition, which resulted in a total weighted sample of 5128. The sample for

86 the survey was selected through a two-stage stratified random sample of households. At the primary stage

87 of sampling, 260 PSUs (82 in urban areas and 178 in rural areas) were selected using systematic sampling

88 with probability proportional to size. At the next stage of sampling, systematic random samples of about

8930 and 36 households per primary sampling unit on the average in urban and in rural areas were selected

90 in all the regions respectively.

\section{Outcome variable}

92 The investigator used NMR as the outcome variable using the recommended definition as children age of

9328 days after birth per 1,000 live births during a given year[20].

\section{Independent variables}

95 The independent variables included in this study were demographic and socioeconomic variables such as

96 (age, religion, region, husband education, wealth status of households, place of residence, sources of

97 drinking water, and availability of toilet facilities), maternal health characteristics like(age at first birth, 
number of children born, previous birth interval, sex of the child, type of birth, place of delivery, mode of

99 delivery, PNC visits) and nutritional assessments like when the child put to breastfed. The current study 100 aimed to women's autonomy effect on neonates, which was leveled based on answered to "Who makes 101 the following decisions in (respondent's) household about 1) obtaining health care for yourself; 2) large 102 household purchases; 3) household purchases for daily needs, and 4) visits to family or relatives?"

103 Response choices were: a) respondent alone; b) respondent and husband/partner; c) respondent and other 104 people; d) husband/partner alone; e) someone else; f) other. The value of 1 is assigned if the response was 105 (a), (b), or (c), that is, the involvement of the respondent, or else 0 , for no involvement of the respondent.

\section{Statistical analysis}

107 For all analyses in this study sampling weight was employed to manage sampling error and non-responses 108 obseravation. Further details on sample weights can be found in the EDHS report[20]. Descriptive 109 statistics were employed to show the distribution of background characteristics. Association between 110 neonatal mortality and the explanatory variables was assessed in bivariate analysis using Chi-square tests.

111 Logistic regression was used to assess the net effect of women's autonomy on neonatal death after 112 controlling several other control variables. Two models were used in the analysis. The first model 113 contained the variables related to women's autonomy. In the second model, other demographic and 114 socioeconomic characteristics were added. Prior to the multivariate analysis, multi-co linearity between 115 the variables was assessed. However, no multi-co linearity was found among the variables. In general all 116 models was performed with those variables showing $P<0.25$ in the unadjusted analysis. Then after, the 117 multivariable model was reduced using the backward stepwise procedure and all the variables in the 118 multivariable model were variables for which $P \leq 0.05$. Both unadjusted and adjusted odds ratios (ORs) 119 were reported with $95 \%$ confidence intervals $(95 \% \mathrm{CI})$. All analyses were performed using statistical 120 software SPSS (version 25). 


\section{Ethics approval}

122 This study is a secondary analysis of publicly available dataset where permission was obtained through 123 registering with the DHS website and therefore no ethics approval was required.

\section{Results}

\section{Socio-demographic characteristics of study participants}

126 A total of 5,128 women were included in this study. The median age of the participants was 31 years. 127 Most of the study participants (48.8\%) were Muslim by religion. The majority (69.2\%) of the participants 128 had no education i.e. more than four out of six mothers. Almost $(84.1 \%)$ of women were rural residents. 129 Regarding the education of the husband (52.2\%) were hadn't education. Almost more than half(53.3\%) of 130 the participants were poor by the combined wealth index of the family. Moreover, around $69.5 \%(3564)$ 131 and $(55.1 \%)$ of the participant were had unimproved drinking water sources and toilet facility respectively 132 [Table 1].

\section{Reproductive history and neonate healthcare service-related characteristics}

134 From the total study participants, almost all (98.3\%) of women had a parity of one, and most(55.4\%) of 135 women gave first birth at the age of 18 to $25 \mathrm{y}$. Two-thirds $(66.2 \%)$ of women gave birth at respondents 136 home for their recent delivery and (46\%) of women had a previous birth interval of $4 y$ and above. Almost 137 more than half $(51.3 \%)$ of neonates were male. Moreover, most $(91.7 \%)$ of women hadn't postnatal 138 checkup within two months for their most recent delivery[Table 1].

\section{Decision-making status of women related characteristics}

140 About $82.1 \%, 75.7 \%$, and $80.3 \%$ of women had involvement in decision making on their own health care,

141 large household purchases, and family or relatives visits respectively[Table 1].

\section{Neonatal mortality Rate}

143 The neonatal mortality rate in the five years preceding the survey was 20.7 deaths per one thousand live 144 births. The NMR varied significantly with different settings. For instance, the NMR was higher among 
illiterate women (15 per 1000 live births) than among literate women (7 per 1000 live births). Similarly, the NMR was significantly higher among women who were not involved in decision making for their own health care (21 per 1000 live births) than among those who were involved in the decision-making process for their own health care (19 per 1000 live births). The NMR was lower among women aged at first birth 26 or more (1 per 1000 live births) than among other age groups (20 per 1000 live births)Via women aged below 18 and 18-25 years. Furthermore, the NMR was lower among women residing in urban(2 per 1000 live births) than among women residing in rural (19 per 1000 live births). The NMR for Muslim women was higher(13per 1000 live births) than among women who had other religious groups (9 per 100 live births). Moreover, the NMR was significantly higher among home-delivered women(13 per 1000 live births) than among those who delivered at health institutions(7per 1000 live births). Unlikely, the NMR was significantly higher for male neonates (14 per 1000 live births) compared to Female neonates (7 per 1000 live births) i.e. the NMR for males was double. Similarly, NMR was significantly higher among those who had multiple births (18 per 1000 live births) than among those who had single births (3 per 1000 live births). The NMR was significantly higher among those who hadn't immediate initiations of breastfed(14 per 1000 live births) than among those who had immediate initiations of breastfed(6 per 1000 live births). The NMR was higher among those who hadn't PNC checkup within two months(21 per 1000 live births) than among neonates who had PNC checkup(1 per1000 live 162 births)[Table 2].

\section{autonomy}

165 From the multivariable logistic regression analysis, women's involvement in their own healthcare-related decision-making autonomy, Place of delivery, age at first birth, neonate sex, Plurality, breastfeeding, and PNC had an association with neonatal death. Those women who hadn't involved in their own healthcarerelated decision-making autonomy were $2.72(\mathrm{AOR}=2.72$; 95\%CI: $1.41,5.24)$ times higher risk of 
171 institutions was $0.61(\mathrm{AOR}=0.61 ; 95 \% \mathrm{CI}: 0.38,0.97)$ times lower than among those women who delivered

172 at their home. Similarly, the death risk of neonate among those women who hadn't PNC checkup was

173 5.48(AOR=0.61; 95\%CI:0.38,0.97) times higher compared to among those women who had PNC.

174 Likewise, those women who had initiation of immediate breastfed were $0.17(\mathrm{AOR}=0.17$;

$17595 \% \mathrm{CI}: 0.12,0.26)$ times lower the death risk of neonate than its counterpart. Moreover, the likelihood of

176 neonate death among those women who had single birth were0.09(AOR=0.09; 95\%CI:0.05,0.18) times

177 lower compared to those women who had multiple births. Unlikely, the death risk of male neonates

178 were1.84(AOR=1.84; 95\%CI:1.20,2.81) times higher than female neonates. The death risk for those

179 women who gave birth of age at first birth 18-25 years were 0.32(AOR=0.32; 95\%CI:0.10,0.99) times

180 lower compared to among those women who gave birth of age at first birth 26 and more[Table 3].

\section{Discussion}

182 Results show that the neonatal mortality rate in Ethiopia was 20.7 per 1000 live births. Thus indicating 183 the situation is alarming the country and need to be addressed by maternal and child health programs. In 184 the present study, the bivariate analysis result shows that women's autonomy (namely, decision making 185 regarding their own health care), Place of delivery, age at first birth, neonate sex, Plurality, breastfeeding, 186 and postnatal care service utilization are important variables in explaining neonatal mortality. The 187 multivariate analysis result confirmed most of the findings of the bivariate analysis. In the multivariate 188 analysis women's autonomy (decision making regarding their own health care), place of delivery, age at 189 first birth, neonate sex, Plurality, breastfeeding, and PNC were significant predictors of neonatal 190 mortality.

192 As do many other studies, this study also shows that neonatal mortality is significantly lower among those 193 mothers who were involved in decisions regarding their own health care compared to those who were not.

194 A possible reason could be that autonomous mothers are more capable of accessing available health 
facilities and that they are able to greatly change the traditional balances of power and autonomy in familial relationships, with profound effects on child care like reduce their reproductive behavior risks,

197 prolong birth intervals, utilize prenatal care, and immunize their children in general and result in lower neonatal mortality. In addition, women can make decisions on delaying the age at first birth which plays a valuable strategy to promote and improve neonatal health and survival. Usually, adolescent mothers face financial and social problems that cause less provision of kid care. Indeed, the physiological immaturity of teenage mothers causes more neonatal death since they may have a small uterus or narrow bony pelvis and lack social experience in caring for newborns. As result empowering, women in decision-making power might be answered all this problem. These findings are consistent with previous studies that assessed the effect of Women's autonomy on maternal and child health service utilization[1, 2, 4-6, 8-10]. Evidence from the previous studies in India has also confirmed that a women's control over household resources (ability to keep money aside) has a significant positive effect on both the demand for prenatal care and the probability of hospital delivery[11].

The death risk proportion of neonates for those whose mother delivered without health facility was higher compared to those whose mother delivered at health institutions. The difference might be happens due to

211 lack of adequate maternal and neonatal care at home. As a result, the newborn baby might not be take the 212 immediate nutrition after birth and it is also difficult to fix complication immediately if there is a problem while the mother delivered at home. These findings are supported by the previous studies that focused on 214 the impact of institutional delivery on maternal health care as a result proper medical attention and 215 hygienic conditions during delivery reduce the occurrence of complications and infections which might 216 be caused to death or serious illness for the mother, baby, or both [3, 6, 15, 16, 19-22]. Indeed, delivered 217 with week health facility like no access of skilled providers, poor infrastructure, and substandard quality 218 of care causes to the risk of intrapartum-related deaths[7, 13]. However, quality of delivery services and 219 variations in newborn care practices weren't included in these analyses but could possibility affect the risk 220 of neonatal deaths. 
221 The sex of the newborn also significantly associated with neonatal mortality. Unknowingly in this study

222 being male are more likely to die than among who are female. Even though, there is no previous study

223 supported the present study the problem might be due to bad cultural practices of the community.

225 The result of this study found that the probability of neonatal mortality is very high in the case of multiple

226 births. This effect is mainly associated with the lower birth weight of twins or triplets, which in turn is

227 one of the most important factors affecting neonatal survival. The reason might be that it needs extra

228 demand for food during the first day of the neonate. Since breastfeeding is one of the main sources of

229 nutrition, multiple births might cause neonates' lower calorie intake, and thus lower survival chances[1,

230 19]. Likewise, breastfeeding is a fundamental concern of proceeding the newborn's life, and the result of

231 this study shown that breastfeeding has a significant positive effect on neonatal mortality. The possible

232 reason might be the deleterious effects of infections related to neonatal deaths can be prevented by breast

233 milk especially practicing breast milk-fed within the first 1hour after birth. This practice not only

234 minimizing the death risk but also helps to wealth and brain development[22].

235

236 The death risk of neonate among those women who hadn't PNC checkup was 5.48 times higher compared

237 to those women who had PNC. This might be the prompt postnatal care (PNC) for both the mother and

238 the child is important to treat any complications arising from the delivery, as well as to provide the 239 mother with important information on how to safeguard herself and her kid. Evidence from the previous 240 studies on safe motherhood programs recommends that all women receive a check of their health within 2 241 days after delivery had a positive effect on the wellbeing of both mothers and neonates[19]. In relation to 242 this, a study in India and rural Sierra Leone indicated that postnatal care has a significant positive effect 243 on neonatal mortality[6,11, 14, 17, 18, 21, 22]. 


\section{Conclusion}

245 In Ethiopia, neonatal mortality was significantly associated with women's hadn't decision making power

246 on health care, hadn't postnatal care, delivered without health institution, not immediately breastfeeding,

247 and gave birth multiple. Hence, it is important to encourage mothers autonomy, access to postnatal care

248 service, and broadened every woman delivery in health institutions in order to reach the Sustainable

249 Development Goals(SDG) of 2030. If programs focus on increasing women's autonomy, neonatal

250 mortality will decrease and the overall well-being of the family will be maintained and be enhanced.

251 Strengths and limitations of the study

252 This study was based on the recent EDHS(2016 )with a nationally representative large sample size.

253 Moreover, this study applied both separated and simultaneously multivariable logistic regression

254 modeling to handle the influence of Women's decision making autonomy alone on neonatal mortality and

255 its associated factors on the basis of the EDHS data. Despite the above strengths, the study might have

256 recall bias since the participants were asked about the events that happened 5 years or more preceding the

257 survey. The study also shares the restrictions of cross-sectional studies.

\section{Abbreviations}

259

AOR

CI

EDHS

HIV/AIDS

NMR

PNC

SDG

SPSS

STI
Adjusted Odds Ratio

Confidence Interval

Ethiopian Demographic and Health Survey

Human Immune Virus

Neonatal Mortality Rate

Postnatal Care

Sustainable Development Goal

Statistical Package for Social Science

Sexually transmitted Infections 


\section{Availability of data and materials}

The data set supporting the conclusions of this article is held by the authors and the Central Statistical Agency, CSA, Ethiopia, and the de-recognized data may be made available if a unique request is crafted from CSA website (https://www.csa.gov.et).

\section{Acknowledgments}

The authors thanks MEASURE DHS for providing access to the data.

\section{Ethics approval and Consent}

Not applicable.

\section{Conflicts of Interest}

The author declare that there is no competing interests.

\section{Funding}

Not applicable.

\section{Authors' Contributions}

HDM developed the idea of the study, acquired the research proposal, carried out statistical analysis and draft the manuscript.

\section{Author's Detail}

Department of Statistics, Collage of Natural and Computational science, Injibara University, Injibara, Amhara, Ethiopia

\section{References}

1. Adhikari, R. and Y. Sawangdee, Influence of women's autonomy on infant mortality in Nepal. Reproductive Health, 2011. 8(1): p. 7.

2. Osamor, P.E. and C. Grady, Women's autonomy in health care decisionmaking in developing countries: a synthesis of the literature. International journal of women's health, 2016. 8: p. 191-202.

3. S, G. and J. H-S, The Role of Women's Autonomy and Experience of Intimate Partner Violence as a Predictor of Maternal Healthcare Service 
Utilization in Nepal. International Journal of Environmental Research and Public Health, 2019. 16(5): p. 895.

4. Jahan, F., S. Hossain, and K. Mahmud, FACTORS INFLUENCING WOMEN'S DECISION MAKING POWER: EVIDENCE FROM BANGLADESH URBAN HEALTH SURVEY DATA. IMPACT: International Journal of Research in Applied, Natural and Social Sciences (IMPACT: IJRANSS), 2015. 3: p. 133-150.

5. Hou, X. and N. Ma, The effect of women's decision-making power on maternal health services uptake: evidence from Pakistan. Health Policy Plan, 2013. 28(2): p. 176-84.

6. Adhikari, R., Effect of Women's autonomy on maternal health service utilization in Nepal: a cross sectional study. BMC women's health, 2016. 16: p. 26-26.

7. Asweto, C., et al., Maternal Autonomy, Distance to Health Care Facility and ANC Attendance: Findings from Madiany Division of Siaya County, Kenya. American Journal of Public Health Research, 2014. 2: p. 153-158.

8. World Health, O., Trends in maternal mortality 2000 to 2017: estimates by WHO, UNICEF, UNFPA, World Bank Group and the United Nations Population Division: executive summary. 2019, World Health Organization: Geneva.

9. UNICEF, W., World Bank and U-DPD, Levels \& Trends in Estimates Developed by the UN Inter-agency Group for Child Mortality Estimation United Nations Levels \& Trends in Child Mortality. 2017.

10. Mekonnen, T., et al., Women's Empowerment as a Determinant of Contraceptive Use in Ethiopia:Further Analysis of the 2011 Ethiopia Demographic and Health Survey D.F.A.R.N. 82, Editor. 2013, Calverton, Maryland, USA: ICF International: ..

11. Maitra, P., Parental bargaining, health inputs and child mortality in India. Journal of Health Economics, 2004. 23(2): p. 259-291.

12. Asweto, C., et al., Maternal Autonomy, Distance to Health Care Facility and ANC Attendance: Findings from Madiany Division of Siaya County, Kenya. American Journal of Public Health Research, 2014. 2(4): p. 153158.

13. Munabi-Babigumira, S., et al., Factors that influence the provision of intrapartum and postnatal care by skilled birth attendants in low-and middle-income countries: a qualitative evidence synthesis. The Cochrane database of systematic reviews, 2017. 11(11): p. CD011558-CD011558. 
14. Cornish, H., et al., Women's economic empowerment and health related decision-making in rural Sierra Leone. Culture, Health \& Sexuality, 2019: p. 1-18.

15. Belachew, A., T. Tewabe, and G. Dessie, Neonatal mortality and its association with antenatal care visits among live births in Ethiopia: a systematic review and meta-analysis. The Journal of Maternal-Fetal \& Neonatal Medicine, 2020: p. 1-8.

16. Kassaw, A., A. Debie, and D.M. Geberu, Quality of Prenatal Care and Associated Factors among Pregnant Women at Public Health Facilities of Wogera District, Northwest Ethiopia. Journal of Pregnancy, 2020. 2020: p. 9592124.

17. Hagaman, A.K., et al., The impacts of quality improvement on maternal and newborn health: preliminary findings from a health system integrated intervention in four Ethiopian regions. BMC Health Services Research, 2020. 20(1): p. 522.

18. (Ethiopia), C.s.a.C. and ICF, Ethiopian demographic and health survey. 2016: Addis Ababa, Ethiopia, and Rockville, Maryland UC and I. No Title.

19. survey, E.m.d.a.h., Ethiopian mini demographic health survey. 2019, Ethiopian public health institute: Addis Ababa, Ethiopia, and Rockville, Maryland UC and I, editor.

20. UNICEF, Neonatal mortality - UNICEF DATA Monitoring the situation of children and women. 2020.

21. Wilunda, C., et al., Evaluation of a maternal health care project in South West Shoa Zone, Ethiopia: before-and-after comparison. Reproductive health, 2016. 13(1): p. 95-95.

22. Memirie, S.T., et al., A cost-effectiveness analysis of maternal and neonatal health interventions in Ethiopia. Health Policy and Planning, 2019.

34(4): p. 289-297. 


\section{Tables}

Table 1: Selected background characteristics of the participants

\begin{tabular}{|c|c|c|c|}
\hline \multicolumn{2}{|l|}{ Characteristics } & $\%$ & $\mathrm{~N}$ \\
\hline \multicolumn{4}{|l|}{ Women's autonomy } \\
\hline \multirow[t]{2}{*}{ Decision on own health care } & Involvement of respondents & 82.1 & 4212 \\
\hline & without involvement of respondents & 17.9 & 916 \\
\hline \multirow{2}{*}{$\begin{array}{l}\text { Decision on making large household } \\
\text { purchases }\end{array}$} & Involvement of respondents & 75.7 & 3882 \\
\hline & without involvement of respondents & 24.3 & 1246 \\
\hline \multirow[t]{2}{*}{ Decision on visits to family or relatives } & Involvement of respondents & 80.3 & 4116 \\
\hline & without involvement of respondents & 19.7 & 1012 \\
\hline \multicolumn{4}{|c|}{ Demographic and socioeconomic characteristics } \\
\hline \multirow[t]{3}{*}{ Age group in years } & $<25$ & 15.1 & 775 \\
\hline & $25-29$ & 30.5 & 1562 \\
\hline & 30 or older & 54.4 & 2791 \\
\hline \multirow[t]{3}{*}{ Religion } & Orthodox & 30.1 & 1541 \\
\hline & Muslim & 48.8 & 2504 \\
\hline & Others & 21.1 & 1083 \\
\hline \multirow[t]{2}{*}{ Residence } & Urban & 15.9 & 816 \\
\hline & Rural & 84.1 & 4312 \\
\hline \multirow[t]{4}{*}{ respondents Highest level of education } & No education & 69.2 & 3550 \\
\hline & Primary & 23.4 & 1200 \\
\hline & Secondary & 5.1 & 259 \\
\hline & Higher & 2.3 & 119 \\
\hline \multirow{4}{*}{$\begin{array}{l}\text { Highest level of husbands/partner } \\
\text { education }\end{array}$} & No education & 52.2 & 2676 \\
\hline & Primary & 32.6 & 1671 \\
\hline & Secondary & 8.8 & 449 \\
\hline & Higher & 6.5 & 332 \\
\hline \multirow[t]{3}{*}{ Wealth index of the family } & Poor & 53.3 & 2734 \\
\hline & Medium & 15.1 & 775 \\
\hline & Rich & 31.6 & 1619 \\
\hline \multirow[t]{2}{*}{ Availability of toilet facility } & No & 44.9 & 2304 \\
\hline & Yes & 55.1 & 2824 \\
\hline
\end{tabular}




\begin{tabular}{|c|c|c|c|}
\hline \multirow[t]{2}{*}{ Source of drinking water } & Unimproved & 69.5 & 3564 \\
\hline & Improved & 30.5 & 1564 \\
\hline \multicolumn{4}{|c|}{ Reproductive history and neonate healthcare service-related characteristics } \\
\hline \multirow[t]{2}{*}{ Place of delivery } & Respondents home & 66.2 & 3396 \\
\hline & Health facility & 33.8 & 1732 \\
\hline \multirow[t]{3}{*}{ Preceding birth interval in years } & $<2$ & 20.6 & 1057 \\
\hline & $2-3$ & 33.3 & 1710 \\
\hline & 4or more & 46.0 & 2361 \\
\hline \multirow[t]{3}{*}{ Age at first birth } & $<18$ & 43.3 & 2218 \\
\hline & $18-25$ & 55.4 & 2842 \\
\hline & $26+$ & 1.3 & 68 \\
\hline \multirow[t]{2}{*}{ Sex of child } & Male & 51.3 & 2631 \\
\hline & Female & 48.7 & 2497 \\
\hline \multirow[t]{2}{*}{ Plurality } & Single & 98.3 & 5042 \\
\hline & Multiple & 1.7 & 86 \\
\hline \multirow[t]{2}{*}{ Breastfeeding status } & Immediate & 71.0 & 3640 \\
\hline & Not immediate & 29.0 & 1488 \\
\hline \multirow[t]{2}{*}{ PNC check up within two months } & No & 91.7 & 4702 \\
\hline & Yes & 8.3 & 426 \\
\hline \multirow[t]{2}{*}{ Neonate status within the 1 st 28 days } & Alive & 97.9 & 5022 \\
\hline & Died & 2.1 & 106 \\
\hline
\end{tabular}


Table 2 neonatal mortality rates (per 1,000 live births) for 5-year periods preceding the survey, by background characteristics $(\mathrm{N}=5,128)$

\begin{tabular}{|c|c|c|}
\hline \multicolumn{2}{|l|}{ Characteristics } & NMR \\
\hline \multicolumn{3}{|l|}{ Women's autonomy } \\
\hline \multirow[t]{2}{*}{ Decision on own health care* } & Involvement of respondents & 19 \\
\hline & without involvement of respondents & 21 \\
\hline \multirow[t]{2}{*}{ Decision on making large household purchases } & Involvement of respondents & 15 \\
\hline & without involvement of respondents & 21 \\
\hline \multirow[t]{2}{*}{ Decision on visits to family or relatives } & Involvement of respondents & 17 \\
\hline & without involvement of respondents & 21 \\
\hline \multicolumn{3}{|l|}{ Demographic and socioeconomic characteristics } \\
\hline \multirow[t]{3}{*}{ Respondents age group in years } & $<25$ & 3 \\
\hline & $25-29$ & 7 \\
\hline & 30 or older & 12 \\
\hline \multirow[t]{3}{*}{ Religion* } & Orthodox & 6 \\
\hline & Muslim & 13 \\
\hline & Others & 3 \\
\hline \multirow[t]{2}{*}{ Residence* } & Urban & 2 \\
\hline & Rural & 19 \\
\hline \multirow[t]{4}{*}{ respondents Highest level of education } & No education & 15 \\
\hline & Primary & 5 \\
\hline & Secondary & 1 \\
\hline & Higher & 1 \\
\hline \multirow[t]{4}{*}{ Highest level of husbands/partner education } & No education & 12 \\
\hline & Primary & 7 \\
\hline & Secondary & 1 \\
\hline & Higher & 1 \\
\hline \multirow[t]{3}{*}{ Wealth index of the family } & Poor & 12 \\
\hline & Medium & 5 \\
\hline & Rich & 3 \\
\hline \multirow[t]{2}{*}{ Availability of toilet facility } & No & 11 \\
\hline & Yes & 10 \\
\hline
\end{tabular}




\begin{tabular}{|c|c|c|}
\hline \multirow[t]{2}{*}{ Source of drinking water } & Unimproved & 15 \\
\hline & Improved & 6 \\
\hline \multicolumn{3}{|c|}{ Reproductive history and neonate healthcare service-related characteristics } \\
\hline \multirow{2}{*}{ Place of delivery* } & Respondents home & 13 \\
\hline & Health facility & 7 \\
\hline \multirow[t]{3}{*}{ Preceding birth interval in years } & $<2$ & 6 \\
\hline & $2-3$ & 6 \\
\hline & 4or more & 9 \\
\hline \multirow[t]{3}{*}{ Age at first birth** } & $<18$ & 11 \\
\hline & $18-25$ & 9 \\
\hline & $26+$ & 1 \\
\hline \multirow[t]{2}{*}{ Sex of child $* * *$} & Male & 14 \\
\hline & Female & 7 \\
\hline \multirow[t]{2}{*}{ Plurality*** } & Single & 18 \\
\hline & Multiple & 3 \\
\hline \multirow[t]{2}{*}{ Breastfeeding status $* * *$} & Immediate & 6 \\
\hline & Not immediate & 14 \\
\hline \multirow[t]{2}{*}{ PNC check up within two months* } & No & 21 \\
\hline & Yes & 1 \\
\hline
\end{tabular}

Note $* * *$ Significant at $\mathrm{p}<0.001 ; * *=\mathrm{p}<0.01$ and $*=\mathrm{p}<0.05$ 
Table 3 Adjusted odds ratios (OR) and 95\% confidence interval (CI) for neonatal mortality within the past five years preceding the survey by selected predictors

\begin{tabular}{|c|c|c|c|c|c|}
\hline \multirow[t]{2}{*}{ Selected predictors } & & \multicolumn{2}{|c|}{ Model I } & \multicolumn{2}{|c|}{ Model II } \\
\hline & & OR & $\mathrm{CI}$ & OR & $\mathrm{CI}$ \\
\hline \multicolumn{6}{|l|}{ Women's autonomy } \\
\hline \multirow[t]{2}{*}{ Decision on own health care } & $\begin{array}{l}\text { Involvement of } \\
\text { respondents }\end{array}$ & 1.00 & & 1.00 & \\
\hline & $\begin{array}{l}\text { without involvement of } \\
\text { respondents(ref.) }\end{array}$ & $1.91 *$ & $1.02,3.59$ & $2.72 * *$ & $1.41,5.24$ \\
\hline \multicolumn{6}{|c|}{ Demographic and socioeconomic characteristics } \\
\hline \multirow[t]{2}{*}{ Residence } & Urban & & & 0.52 & $0.23,1.20$ \\
\hline & Rural(ref.) & & & 1.00 & \\
\hline \multirow{3}{*}{$\begin{array}{l}\text { Respondents age group in } \\
\text { years }\end{array}$} & $<25$ & & & 0.61 & $0.31,1.19$ \\
\hline & $25-29$ & & & 1.00 & $0.63,1.59$ \\
\hline & 30 or older(ref.) & & & 1.00 & \\
\hline \multirow[t]{3}{*}{ Religion } & Orthodox & & & 1.19 & $0.57,2.45$ \\
\hline & Muslim & & & 1.94 & $0.99,3.77$ \\
\hline & others(ref.) & & & 1.000 & \\
\hline \multirow{4}{*}{$\begin{array}{l}\text { Highest level of } \\
\text { husbands/partner education }\end{array}$} & No education & & & 1.52 & $0.49,4.72$ \\
\hline & Primary & & & 1.65 & $0.53,5.15$ \\
\hline & Secondary & & & 0.77 & $0.18,3.25$ \\
\hline & Higher(ref.) & & & 1.00 & \\
\hline \multirow[t]{3}{*}{ Wealth index of the family } & Poor & & & 0.83 & $0.44,1.54$ \\
\hline & Medium & & & 1.03 & $0.52,2.04$ \\
\hline & Rich(ref.) & & & 1.00 & \\
\hline \multirow[t]{2}{*}{ Availability of toilet facility } & No & & & 1.12 & $0.68,1.84$ \\
\hline & Yes(ref.) & & & 1.00 & \\
\hline \multirow[t]{2}{*}{ Source of drinking water } & Unimproved & & & 0.70 & $0.45,1.11$ \\
\hline & Improved(ref.) & & & 1.00 & \\
\hline \multicolumn{6}{|c|}{ Reproductive history and neonate healthcare service-related characteristics } \\
\hline \multirow[t]{2}{*}{ Place of delivery } & Health facility & & & $0.61 *$ & $0.38,0.97$ \\
\hline & Respondents home & & & 1.00 & \\
\hline
\end{tabular}




\begin{tabular}{|c|c|c|c|c|}
\hline \multirow{3}{*}{$\begin{array}{l}\text { Preceding birth interval in } \\
\text { years }\end{array}$} & $<2$ & & 1.62 & $0.95,2.74$ \\
\hline & $2-3$ & & 1.08 & $0.66,1.77$ \\
\hline & 4or more(ref.) & & 1.00 & \\
\hline \multirow[t]{3}{*}{ Age at first birth } & $<18$ & & 0.48 & $0.16,1.49$ \\
\hline & $18-25$ & & $0.32 *$ & $0.10,0.99$ \\
\hline & $26+$ (ref.) & & 1.00 & \\
\hline \multirow[t]{2}{*}{ Sex of child } & Male & & $1.84 * *$ & $1.20,2.81$ \\
\hline & Female(ref.) & & 1.00 & \\
\hline \multirow[t]{2}{*}{ Plurality } & Single & & $0.09 * * *$ & $0.05,0.18$ \\
\hline & Multiple(ref.) & & 1.00 & \\
\hline \multirow[t]{2}{*}{ Breastfeeding status } & Immediate & & $0.17 * * *$ & $0.12,0.26$ \\
\hline & Not immediate(ref.) & & 1.00 & \\
\hline \multirow{2}{*}{$\begin{array}{l}\text { PNC check up within two } \\
\text { months }\end{array}$} & No & & $5.48 *$ & $1.29,23.26$ \\
\hline & Yes(ref.) & & 1.00 & \\
\hline \multicolumn{2}{|l|}{ Constant } & $0.014 * * *$ & \multicolumn{2}{|l|}{$0.05^{*}$} \\
\hline \multicolumn{2}{|l|}{-2log likelihood } & 1026.77 & \multicolumn{2}{|l|}{861.51} \\
\hline \multicolumn{2}{|l|}{ Cox \& Snell R square } & 0.001 & \multicolumn{2}{|l|}{0.033} \\
\hline
\end{tabular}

Note $* * *$ Significant at $\mathrm{p}<0.001 ; * *=\mathrm{p}<0.01$ and $*=\mathrm{p}<0.05$ 